\title{
Quantification of the Impact of Partition Coefficient Prediction Methods on Physiologically Based Pharmacokinetic Model Output Using a Standardized Tissue Composition ${ }^{\mathbb{}}$
}

\author{
Kiersten Utsey, Madeleine S. Gastonguay, Sean Russell, Reed Freling, Matthew M. Riggs, \\ and $\odot$ Ahmed Elmokadem
}

Metrum Research Group, Tariffville, Connecticut (K.U., M.S.G., S.R., R.F., M.M.R., A.E.); University of Utah, Salt Lake City, Utah (K.U.); University of Connecticut, Storrs, Connecticut (M.S.G.); University of Michigan, Ann Arbor, Michigan (S.R.); and Cornell University, Ithaca, New York (R.F.)

Received January 17, 2020; accepted July 6, 2020

\begin{abstract}
Tissue:plasma partition coefficients are key parameters in physiologically based pharmacokinetic (PBPK) models, yet the coefficients are challenging to measure in vivo. Several mechanistic-based equations have been developed to predict partition coefficients using tissue composition information and the compound's physicochemical properties, but it is not clear which, if any, of the methods is most appropriate under given circumstances. Complicating the evaluation, each prediction method was developed, and is typically employed, using a different set of tissue composition information, thereby making a controlled comparison impossible. This study proposed a standardized tissue composition for humans that can be used as a common input for each of the five frequently used prediction methods. These methods were implemented in $\mathbf{R}$ and were used to predict partition coefficients for 11 drugs, classified as strong bases, weak bases, acids, neutrals, and zwitterions. PBPK models developed in R (mrgsolve) for each drug and each set of partition coefficient predictions were compared with respective observed plasma
\end{abstract}

concentration data. Percent root mean square error and half-life percent error were used to evaluate the accuracy of the PBPK model predictions using each partition coefficient method as summarized by strong bases, weak bases, acids, neutrals, and zwitterions characterization. The analysis indicated that no partition coefficient method consistently yielded the most accurate PBPK model predictions. As such, PBPK model predictions using all partition coefficient methods should be considered during drug development.

\section{SIGNIFICANCE STATEMENT}

Several mechanistic-based methods exist to predict tissue:plasma partition coefficients critical to PBPK modeling. Controlled comparisons are confounded by the use of different tissue composition values for each method; a standardized tissue composition was proposed. Resulting assessments indicated that no method was consistently superior; therefore, sensitivity of PBPK predictions to each method may be warranted prior to model optimization.

\section{Introduction}

Physiologically based pharmacokinetic (PBPK) models predict the absorption, distribution, metabolism, and excretion properties of a drug at physiologically relevant (e.g., tissue and organ) scales. Since PBPK models are based on first principles, they can be used to make pharmacokinetic (PK) predictions for the drug of interest prior to conducting clinical trials. Common applications include first-in-human, environmental toxicology, or rare-disease populations studies (Jones and Rowland-Yeo, 2013). These models combine physiologic data with

This work received no external funding.

Portions of this work were previously presented: Utsey K, Gastonguay MS, Russell S, Freling R, Riggs MM, and Elmokadem A. Impact of partition coefficient prediction methods on PBPK model output using a unified tissue composition, in ACoP10; 2019; Orlando, FL. ISSN:2688-3953, Vol 1.

https://doi.org/10.1124/dmd.120.090498.

S This article has supplemental material available at dmd.aspetjournals.org. drug physicochemical properties to parameterize ordinary differential equations that represent the absorption, distribution, metabolism, and excretion processes. Among the drug-related parameters are the tissue: plasma partition coefficients $\left(K_{p}\right)$. In vivo experiments that measure tissue and plasma drug concentrations over time and at steady state can determine $K_{p}$ values; however, these experiments are expensive and time-consuming (Jones and Rowland-Yeo, 2013). As such, these experiments cannot be used for routine data collection in drug discovery. Several in silico methods have been developed to predict $K_{p}$ values from more easily obtained in vitro data. Using a combination of tissue composition information and the compound's physicochemical characteristics, such as lipophilicity $(\log P)$ and the unbound fraction in plasma $\left(f u_{p}\right)$, these methods account for the distribution of the drug between water and drug-binding components, including proteins, lipids, and phospholipids.

Each method assumes that drugs are distributed homogeneously into plasma and each tissue via passive diffusion. They also account for nonspecific binding to tissue components, including lipids, phospholipids,

ABBREVIATIONS: AR, albumin ratio; Berez, Berezhkovskiy prediction method; $f u_{p}$, unbound fraction in plasma; log $P$, lipophilicity; LR, lipoprotein ratio; $K_{p}$, tissue:plasma partition coefficients; PBPK, physiologically based pharmacokinetic; PCC, Pearson correlation coefficient; PK, pharmacokinetic; PK-Sim, default PK-Sim prediction method; PT, Poulin and Theil prediction method; RMSE, root mean square error; RR, Rodgers and Rowland prediction method; Schmitt, Schmitt prediction method. 
and proteins. Although each prediction method is based on the same general mechanisms, the methods differ in complexity and the type of experimental information required. Poulin and Theil (PT) initially proposed a tissue:plasma partition coefficient prediction method that accounts for dissolution into water and nonspecific binding to neutral lipids and phospholipids (Poulin and Theil, 2002). Lipophilicity was estimated by the octanol:water partition coefficient for nonadipose tissue and the oil: water partition coefficient for adipose. Berezhkovskiy (Berez) modified the PT method and assumed only drugs in the water fraction bind to tissues (Poulin and Theil, 2002; Berezhkovskiy, 2004). Rodgers and Rowland (RR) extended Poulin and Theil's method to consider the impact of drug ionization on partitioning (Rodgers et al., 2005; Rodgers and Rowland, 2006). In particular, the new equations in RR accounted for the dissolution of the drug into water; partitioning of un-ionized drugs into neutral lipids and phospholipids; electrostatic interactions between moderate-to-strong bases and acidic phospholipids; and interactions between extracellular proteins and weak bases, acids, neutrals, and zwitterions. Schmitt considered a universal method that separated tissues into water, neutral lipids, neutral and acidic phospholipids, and protein fractions (Schmitt, 2008). Unlike the previous methods, Schmitt accounted for electrostatic interactions between positively charged molecules and acidic phospholipids. Willmann et al. (2005) (PK-Sim) proposed a method that considered partitioning into lipids, proteins, and water and used membrane affinity as a lipophilicity measure. Other empirical methods requiring in vivo data, such as the volume of distribution or the partition coefficient for one tissue, have been proposed (Arundel, 1997; Jansson et al., 2008; Poulin and Theil, 2009). This study considers only commonly used, mechanistic-based methods that require only in vitro data.

Given the wide variability in $K_{p}$ predictions between these different in silico methods, Graham et al. (2012) were motivated to compare the predictive performance between three mechanistic methods (PT, Berez, and RR) and three empirical methods to determine the most accurate method for rat partition coefficients. The study found that among predictions made by the mechanistic-based methods and empirical methods, the predictions made by RR were most accurate in comparison with experimentally determined $K_{p}$ values (Graham et al., 2012). That study only considered $K_{p}$ predictions for rat tissues, however, and was not extended to the human physiology, which is often markedly different from rats (Graham et al., 2012). Additionally, the rat tissue composition used was the one reported by Rodgers and Rowland, which might have led to some bias toward that method. Given the limited availability of experimentally measured human partition coefficients, this study described herein uses a different strategy to compare partition coefficient predictions from different methods.

The goal of the current study was to investigate the impact of five commonly used tissue:plasma partition coefficient prediction methods (PT, Berez, RR, Schmitt, and PK-Sim) on PBPK model predictions based on a standardized human physiology and the physicochemical properties of 11 distinct drugs.

\section{Methods}

Overview. The workflow for the analyses described herein followed a five-step approach (Fig. 1). The first two steps involved the recapitulation of the mathematical expressions used in the published $K_{p}$ estimation equations into $\mathrm{R}$ functions with subsequent reviews to ensure their proper translation and application of these estimation methods. The third and fourth steps involved the development and qualification of a standardized tissue composition data base to act as a control set in the underlying PBPK model while investigating the impact of the different $K_{p}$ estimation methods. The final stage integrated the previous steps to then evaluate each $K_{p}$ estimation method using drugs that were representative of a range of physicochemical properties (strong base, weak base, acid, neutral, and zwitterion). Additional methodology for each step is provided in the sections below.

Tissue:Plasma Partition Coefficient Calculation Methods. Five of the most widely used tissue:plasma partition coefficient methods were included in this investigation (Poulin and Theil, 2002; Berezhkovskiy, 2004; Rodgers et al., 2005; Willmann et al., 2005; Rodgers and Rowland, 2006; Schmitt, 2008). All of these methods require tissue composition data and physicochemical drug properties as inputs, but they vary in type and quantity of data. Some of the methods have multiple equations to account for different classes of drugs or types of tissues.

Poulin and Theil Method. The PT method uses drug solubility and the binding of the drug to macromolecules to predict tissue:plasma partition coefficients (Poulin and Theil, 2002). Equation 1a gives the partition coefficient for nonadipose tissue:

$$
K_{p}=\frac{P_{o: w}\left(f_{n l t}+0.3 f_{p h t}\right)+\left(f_{w t}+0.7 f_{p h t}\right)}{P_{o: w}\left(f_{n l p}+0.3 f_{p h p}\right)+\left(f_{w p}+0.7 f_{p h p}\right)} \frac{f u_{p}}{f u_{t}}
$$

where $P_{o: w}$ is the $n$-octanol:buffer partition coefficient of the nonionized species at $\mathrm{pH} 7.4, f_{n l}$ is the fractional volume of neutral lipids, $f_{p h}$ is the fractional volume of phospholipids, $f_{w}$ is the fractional volume of water, and $f u$ is the unbound fraction of drug. The subscripts $t$ and $p$ indicate tissue and plasma, respectively. Equation $1 \mathrm{~b}$ gives the adipose partition coefficient:

$$
K_{p}=\frac{D_{o: w}^{*}\left(f_{n l t}+0.3 f_{p h t}\right)+\left(f_{w t}+0.7 f_{p h t}\right)}{D_{o: w}^{*}\left(f_{n l p}+0.3 f_{p h p}\right)+\left(f_{w p}+0.7 f_{p h p}\right)} \frac{f u_{p}}{1}
$$

where $D_{o: w}^{*}$ is the olive oil:buffer partition coefficient of both the nonionized and ionized species at $\mathrm{pH}$ 7.4. Poulin and Theil had previously demonstrated that $D_{o: w}^{*}$ yields a better prediction for adipose tissue partition coefficients (Poulin et al., 2001). Further, $f u_{t}$ is set to one because macromolecular binding is negligible in adipose tissue. Poulin and Theil reported the steady-state volume of distribution, $V_{s s}$, rather than $K_{p}$ values. $V_{s s}$ is given by

$$
V_{s s}=\left(\sum V_{t} K_{p}\right)+V_{e} E: P+V_{p}
$$

where $V$ is the fractional body volume of a tissue $(t)$, erythrocyte $(e)$, and plasma $(p)$, and $E: P$ is the erythrocyte:plasma ratio. $E: P$ is estimated as

$$
E: P=\frac{B P-(1-H t)}{H t}
$$

where $B P$ is the in vitro blood:plasma ratio, and $H t$ is the hematocrit content in blood, assumed to be $45 \%$.

Berezhkovskiy Method. Berezhkovskiy derived a modified version of the PT method that does not require the assumption

$$
\frac{f_{w t}+0.7 f_{p h t}}{f_{w p}+0.7 f_{p h p}}=1
$$

but instead only considers tissue binding in the water fraction according to the following equations (Berezhkovskiy, 2004):

$$
\begin{gathered}
K_{p}=\frac{P_{o: w}\left(f_{n l t}+0.3 f_{p h t}\right)+\frac{f_{w t}}{f u_{t}}+0.7 f_{p h t}}{P_{o: w}\left(f_{n l p}+0.3 f_{p h p}\right)+\frac{f_{w p}}{f u_{p}}+0.7 f_{p h p}} \\
K_{p}=\frac{D_{o: w}^{*}\left(f_{n l t}+0.3 f_{p h t}\right)+\frac{f_{w t}}{f u_{t}}+0.7 f_{p h t}}{D_{o: w}^{*}\left(f_{n l p}+0.3 f_{p h p}\right)+\frac{f_{w p}}{f u_{p}}+0.7 f_{p h p}}
\end{gathered}
$$


1. Create $\mathbf{R}$ functions to calculate $K_{p}$ estimates

- Use mathematical formula reported for the $5 K_{p}$ estimation methods (PT, Berez, RR, Schmitt, and PK-Sim)

- Use respective inputs for each method specific to each drug evaluated

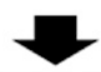

2. Validation and verification of the new $\mathrm{R}$ functions for $K_{p}$ estimation

- Compare calculated $K_{p}$ values from the newly generated functions, for different drug classes, to the reported values

- Use the reported tissue compositions used for each separate $K_{p}$ estimation method

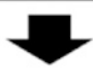

3. Develop a standardized tissue composition database

- Use of human values, reported across several sources, was preferred

- Values from nonclinical sources (e.g., rats) used only when corresponding values were not available

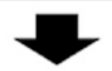

4. Validation and verification of standardized tissue compositions

- Compare predicted $K_{p}$ values using the standardized to the $K_{p}$ values using the separately reported tissue compositions

Fig. 1. The analysis workflow.

$$
K_{p}=\left(f_{e w}+\frac{1+X}{1+Y} f_{i w}+\frac{K a_{a p} f_{a p l} X}{1+Y}+\frac{P f_{n l}+(0.3 P+0.7) f_{n p l}}{1+Y}\right) f u_{p}
$$

where $f_{e w}$ is the fraction of extracellular water, $f_{i w}$ is the fraction of intracellular water, $f_{a p l}$ is the fraction of acidic phospholipids, $f_{n l}$ is the fraction of neutral lipids, $f_{n p l}$ is the fraction of neutral phospholipids, $K a_{a p}$ is the association constant between the drug and acidic phospholipids, and $P$ is the $n$-octanol:buffer partition coefficient for nonadipose tissue and the olive oil:buffer partition coefficient for adipose tissue. $X$ and $Y$ are the ionization terms for the drug in intracellular water and plasma, respectively, and were calculated using the HendersonHasselbalch equation (Radić and Prkić, 2012). In the case of a monoprotic base, for example, $X=10^{\mathrm{p} K_{a}-\mathrm{pH}_{i w}}$ and $Y=10^{\mathrm{p} K_{a}-\mathrm{pH}_{p}}$, where $\mathrm{pH}_{i w}$ is the $\mathrm{pH}$ of intracellular water and $\mathrm{pH}_{p}$ is the $\mathrm{pH}$ of plasma.

The equation for acids, very weak bases, neutrals, and zwitterions with $\mathrm{p} K_{a}<7$ incorporated partitioning into neutral lipids and phospholipids, the dissolution of the drug into tissue water, and associations with extracellular proteins. The partition coefficients are given by

$$
K_{p}=\left(f_{e w}+\frac{1+X}{1+Y} f_{i w}+\frac{K a_{p r} f_{p r} X}{1+Y}+\frac{P f_{n l}+(0.3 P+0.7) f_{n p l}}{1+Y}\right) f u_{p}
$$

where $f_{p r}$ is the fraction of albumin for acids, very weak bases, and zwitterions with $\mathrm{p} K_{a}<7$ and the fraction of lipoprotein for neutral drugs.

Schmitt Method. The Schmitt method explicitly considered the electrostatic interactions between charged molecules at physiologic $\mathrm{pH}$ and acidic phospholipids (Schmitt, 2008). The lipid subcompartment consisted of neutral lipids, neutral phospholipids, and acidic phospholipids. The partition coefficients are given by

$$
K_{p}=\left(f_{w}+K_{n l} f_{n l}+K_{n p l} f_{n p l}+K_{a p l} f_{a p l}+K_{p r} f_{p r}\right) f u_{p}
$$

where $K_{n p l}$ is the neutral phospholipids:water partition coefficient, $K_{a p l}$ is the acidic phospholipid:water partition coefficient, and $K_{p r}$ is the protein:water partition coefficient.

PK-Sim Standard Method. PK-Sim software, which is part of the Open Systems Pharmacology Suite (http://www.open-systemspharmacology.org/; ()Lippert et al., 2019), adopted a default partition coefficient calculation method proposed by Willmann et al. (2005). This method incorporates partitioning into tissue water, lipids, and proteins, where $K_{p}$ is calculated as:

$$
K_{p}=\left(f_{w}+K_{\text {lipid }} f_{\text {lipid }}+K_{p r} f_{p r}\right) f u_{p} .
$$

All five methods were implemented as $\mathrm{R}$ script functions ( $\mathrm{R}$ Core Team, 2018) whose outputs were verified against partition coefficients 
reported in the corresponding papers (see associated Github repository https://github.com/metrumresearchgroup/PBPK_PC). Pearson correlation coefficients (PCCs) were calculated using predictions for strong bases, weak bases, acids, neutrals, and zwitterions. The papers reported predicted coefficients for different drugs, so the particular drugs used in the PCC calculation varied. Berezhkovskiy, Schmitt, and Willmann et al. did not report predicted partition coefficients or $V_{s s}$ values. In these cases, PK-Sim software outputs for each of the three methods were used for verification.

To investigate the impact of different tissue compositions on predictions, $K_{p}$ predictions from PT, Berez, Schmitt, and PK-Sim, using two drugs from each class (metoprolol, acebutolol-R, voriconazole, alprazolam, thiopental, phenobarbital, digoxin, ethoxybenzamide, ofloxacin, and enoxacin) and the originally reported respective tissue compositions, were compared against the same predictions from the RR reported tissue composition, and PCCs were calculated. The RR reported tissue composition was the only one that could be swapped with inputs of other methods because it alone included all of the necessary parameters for the remaining four methods.

Development of a Standardized Tissue Composition Data Base. Each tissue:plasma partition coefficient method included in this study used a different set of tissue composition information. Whereas some of the methods used tissue composition for humans, other methods, such as $\mathrm{RR}$, used tissue composition information from rats. Differences in predictions from the methods could have been due to both the tissue composition and the methods themselves, thereby confounding comparisons of the predictions from the five partition coefficient methods.

Since tissue composition influences tissue:plasma partition coefficient predictions, this study proposed a standardized tissue composition that can be used with each of the partition coefficient methods. The standardized tissue composition combined information from several sources to avoid biasing the evaluation of the partition coefficient methods (Open Systems Pharmacology; Poulin and Theil, 2002; Rodgers et al., 2005; Rodgers and Rowland, 2006; Ruark et al., 2014). Human values for certain types of tissue composition were not found in literature, so values for rats were used in the standardized tissue composition. In particular, the $f_{e w}, f_{i w}, \mathrm{pH}$, albumin ratio (AR), and lipoprotein ratio (LR) for all tissues, and $f_{n p l}$ for bone and gut, were from rats (Rodgers et al., 2005; Rodgers and Rowland, 2006). It was assumed that $f_{\text {water }}=f_{\text {ew }}+f_{i w}$ for all tissues except plasma and red blood cells, which do not have $f_{e w}$, and for these, $f_{\text {water }}$ values were taken from Ruark et al. (2014). The standardized tissue composition was used as input for each partition coefficient method. Partition coefficient predictions from each method using the standardized tissue composition were compared with predictions from the same method using the corresponding reported tissue compositions. The PCC was calculated for each method using partition coefficients for all tissues for the same drugs used in the comparison in the previous section.

In addition to the analysis comparing the PBPK predictions using the standardized tissue composition, a parallel analysis was carried out using the originally reported tissue compositions for each of the calculation methods. This was done to evaluate bias possibly associated with any one of the methods that could arise from using the standardized tissue composition data base.

PBPK Model Framework. The PBPK model used ordinary differential equations to describe well mixed tissues that were linked by the blood system. The model assumed perfusion rate-limited kinetics. A PBPK model including 15 tissue compartments was implemented for each drug in this study. The body for this model was composed of the following tissues: lung, adipose, bone, brain, heart, kidney, muscle, skin, liver, pancreas, spleen, and gut (Fig. 2). The model also included compartments for venous blood and arterial blood, and

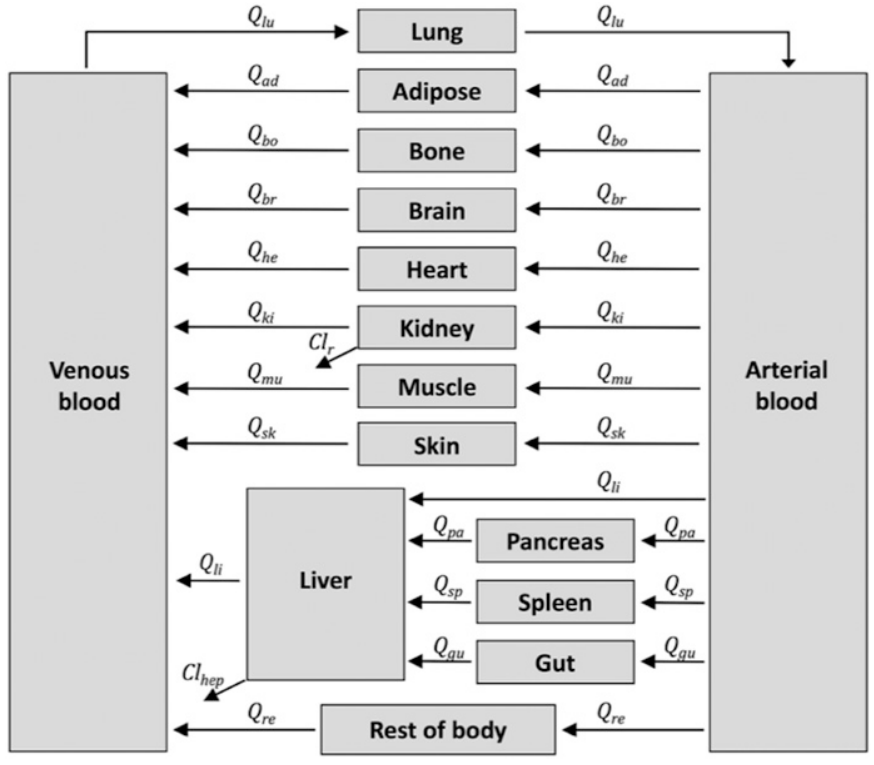

Fig. 2. Schematic for the general, flow-limited, physiologically based pharmacokinetic model. The drug can be cleared from the kidney and liver compartments. Tissues that are not explicitly included in the model are represented by the rest-ofbody compartment. Q refers to blood flow with the subscripts $a d, b o, b r, h e, k i, m u$, $l i, l u, s k, p a, s p, g u$, and $r e$ refer to adipose, bone, brain, heart, kidneys, muscle, liver, lungs, skin, pancreas, spleen, gut, and rest of body, respectively. $\mathrm{Cl}_{h e p}$ and $C l_{r}$ refer to hepatic and renal clearances, respectively.

a "rest-of-body" compartment that represented the remainder of the tissues not explicitly included in the model. The volume of the rest-ofbody compartment was derived by subtracting the volumes of all other compartments from body weight, whereas the blood flow to this compartment was derived by subtracting the blood flows of all compartments entering into venous blood from the cardiac output. The model equations are discussed further in the Supplemental Information section. Model parameters, including tissue volumes and blood flows, body weight, cardiac output, and drug-specific parameters, are also in the Supplemental Information section (Supplemental Tables 1 and 2).

The general PBPK model was implemented in $\mathrm{R}$ by using the opensource package mrgsolve ((R Core Team, 2018); Elmokadem et al., 2019; Gastonguay et al., 2019). Tissue:plasma partition coefficients were predicted for the tested drugs using each method, and each set of partition coefficients was used as input in the PBPK model, resulting in five PBPK model predictions for each tested drug. The rest-of-body partition coefficient was calculated as the average of the nonadipose tissue:plasma partition coefficients. The PBPK model was used to predict plasma concentration profiles for $11 \mathrm{drugs}$, and the predictions were compared with experimental data. The general PBPK model framework was modified for each drug to account for differences in administration route and clearance. Additional details about the model are described in the Supplemental Information section.

Drugs Included in the Study. Drugs were divided into five classes: strong bases, weak bases, acids, neutrals, and zwitterions. The division was made based on how the tissue:plasma partition coefficient methods account for differences in drugs. For example, RR accounts for different types of interactions between tissue components and strong and weak bases, but it assumes that strong and weak acids have the same types of interactions with tissue components (Rodgers et al., 2005; Rodgers and Rowland, 2006). Drugs were selected based on availability of published observed plasma concentrations and physicochemical parameters, including $\log P, \mathrm{p} K_{a}, B P, f u_{p}$, clearance rates, and absorption rates. The strong bases investigated were metoprolol and caffeine, and the weak 
A

A PT

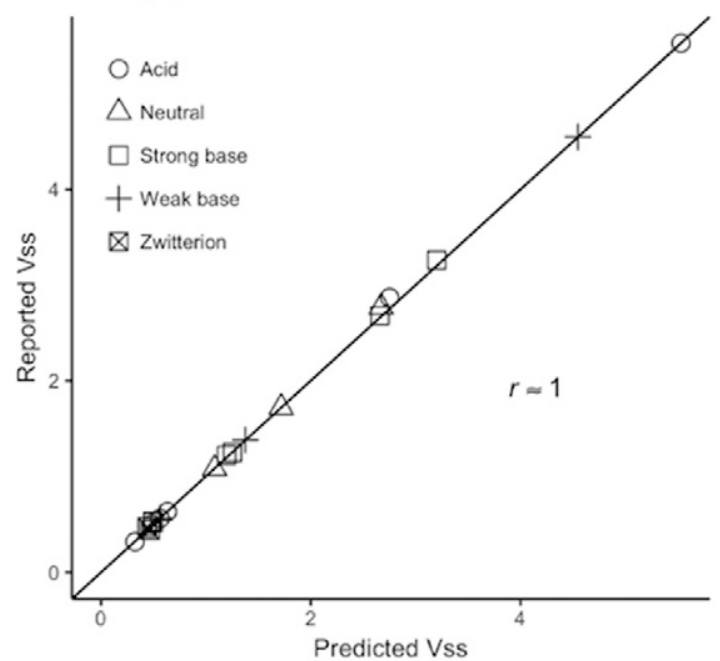

C

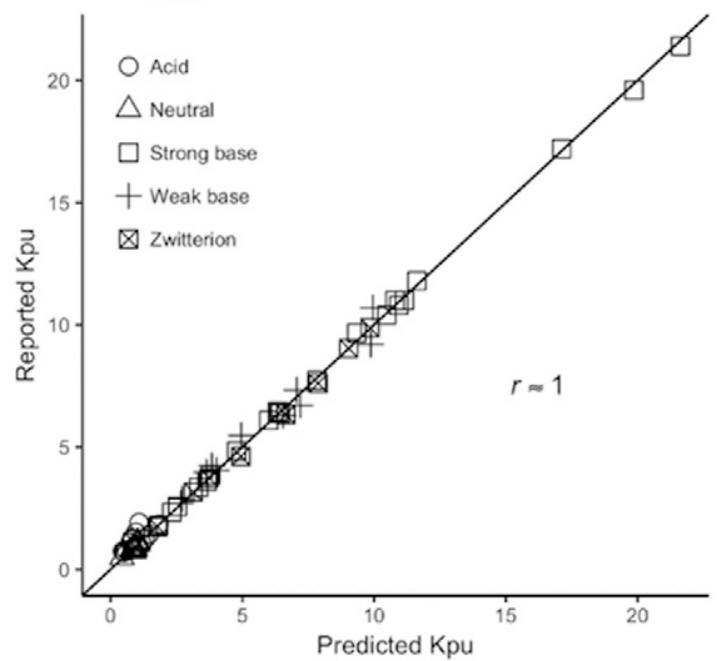

B Berez

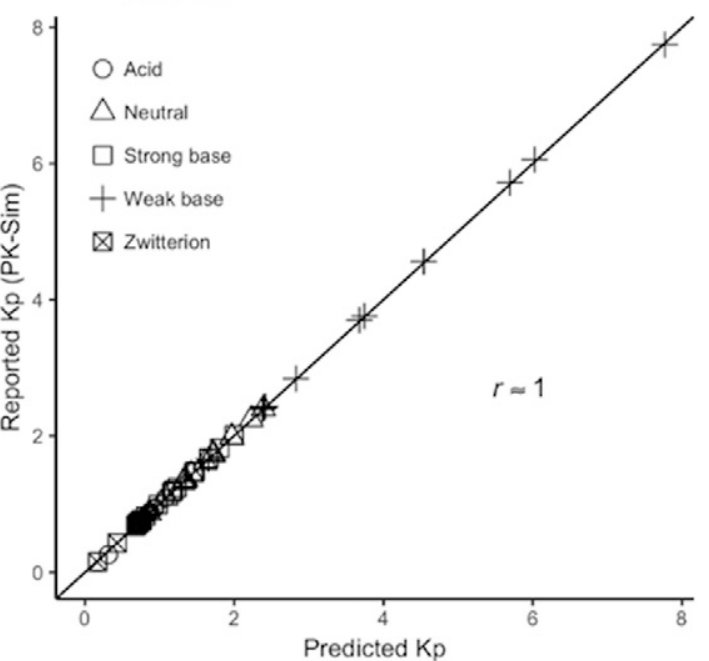

D Schmitt

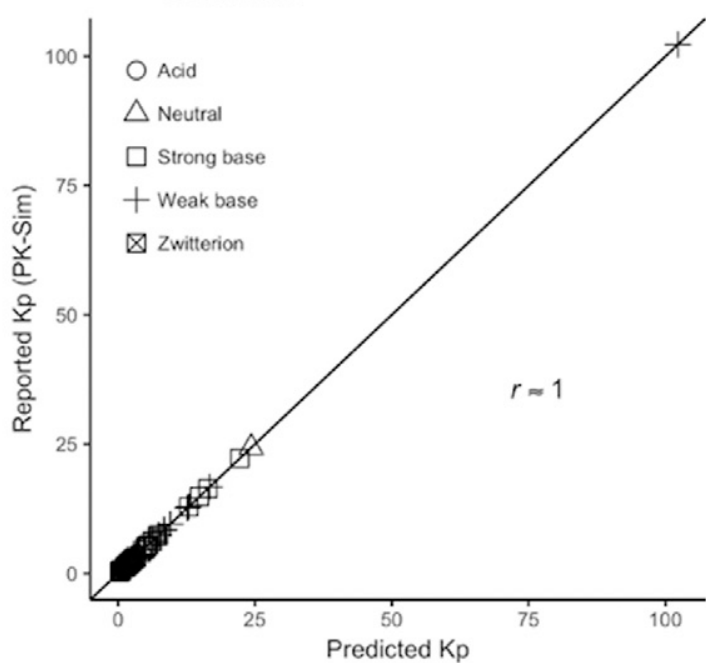

E PK-Sim

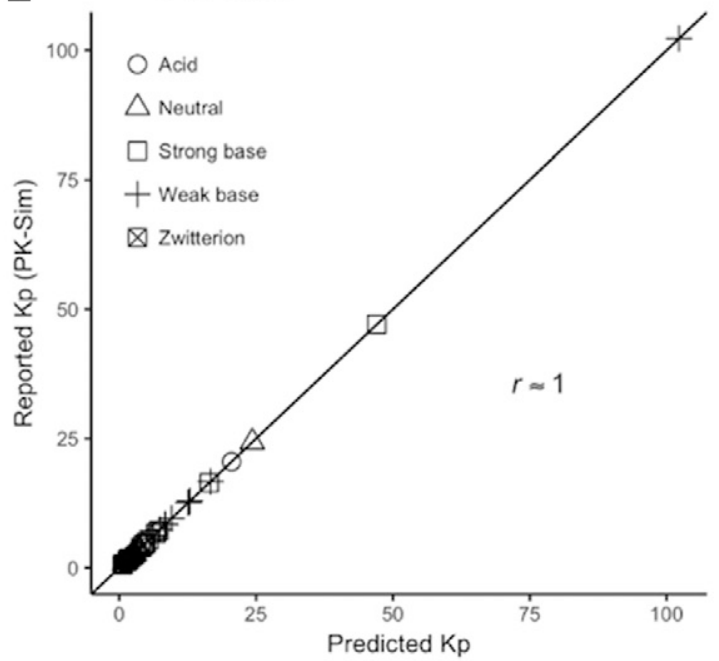

Fig. 3. Verification of the open-source R script predictions with reported prediction values for each method. The PCC for each method is denoted by $r$. (A) Comparison between $V_{s s}$ values calculated from the PT $K_{p}$ values and the reported steady-state volume of distribution $\left(V_{s s}\right)$ predictions. (B) Comparison between $K_{p}$ values calculated by the Berez script and $K_{p}$ values predicted using the Berez method within PK-Sim. (C) Comparison between the unbound tissue:plasma water partition coefficient $\left(K_{p u}\right)$ predictions from the RR script and the reported $K_{p u}$ values. (D) Comparison between $K_{p}$ values from the Schmitt script and $K_{p}$ values predicted using the Schmitt method within PK-Sim. (E) Comparison between $K_{p}$ values from the PK-Sim script and $K_{p}$ values predicted using the PK-Sim method within PK-Sim. The scripts reproduce partition coefficient predictions for all five methods. 
A

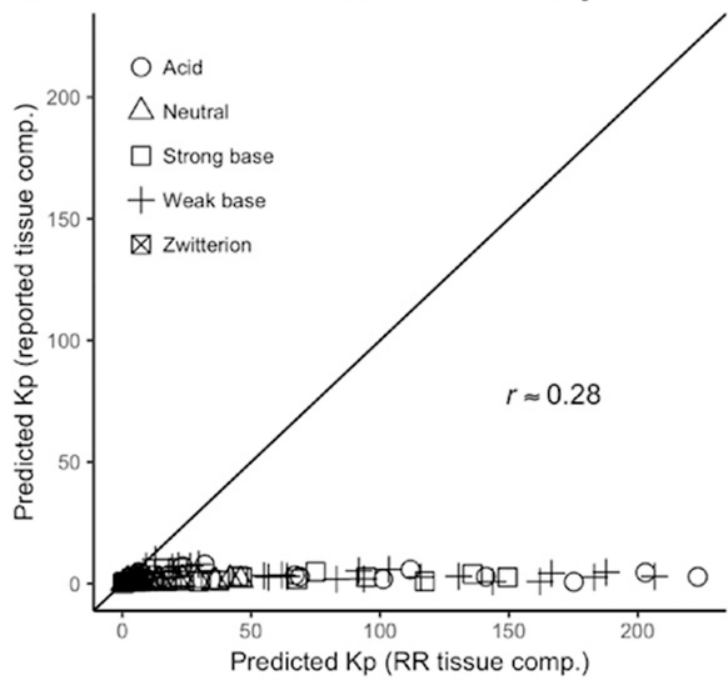

C

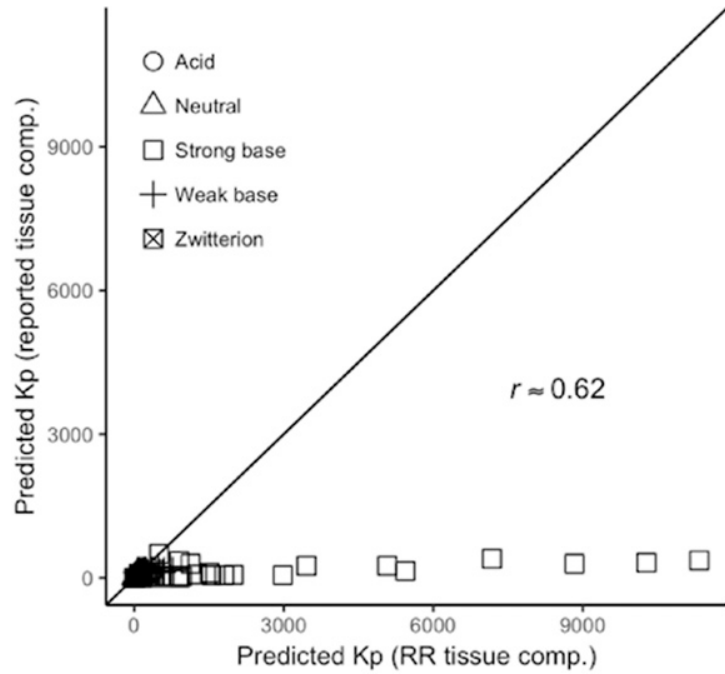

B Berez with RR tissue comp.

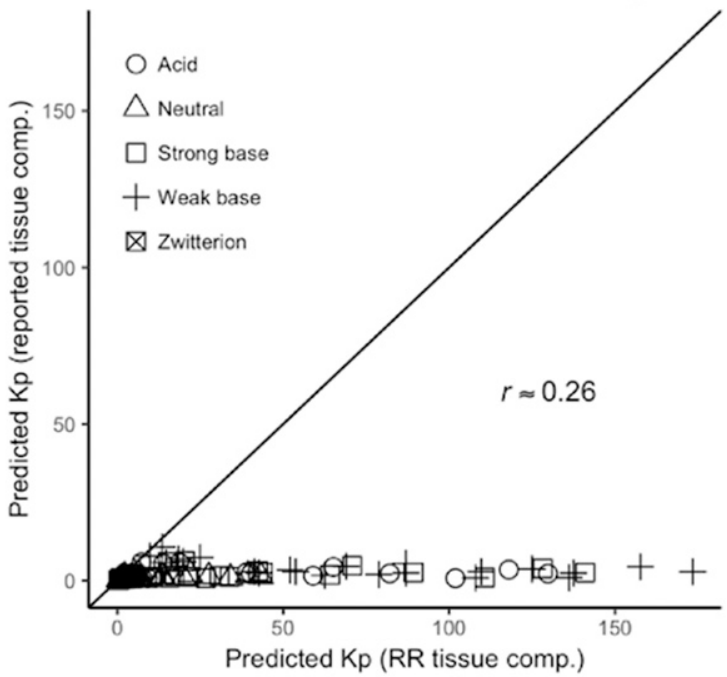

PK-Sim with RR tissue comp.

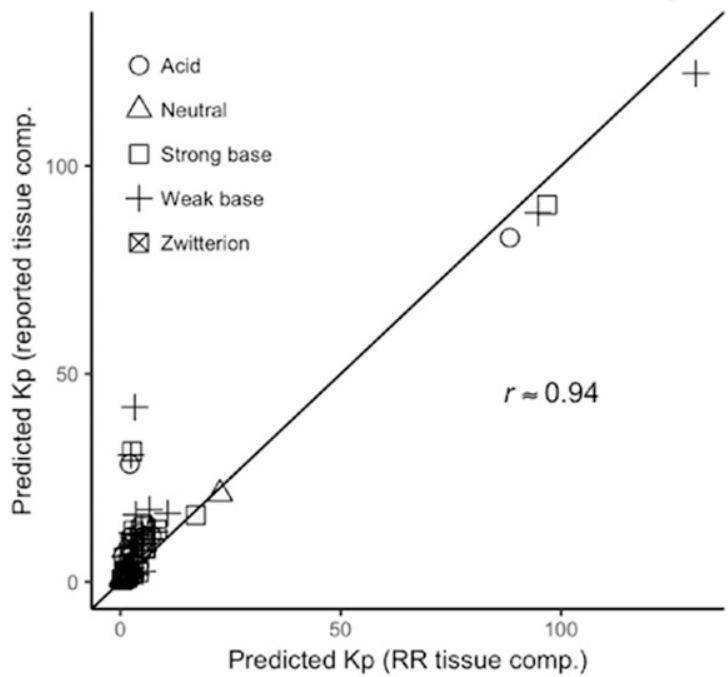

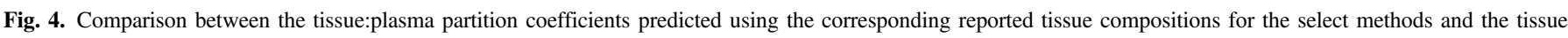

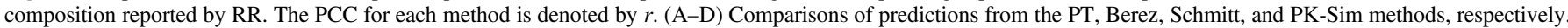
Predictions from each method are impacted by the use of different tissue compositions.

bases were voriconazole, alfentanil, nevirapine, and midazolam (Björkman et al., 1998; Gaohua et al., 2012; Zane and Thakker, 2014; De Sousa Mendes et al., 2017; Elmokadem et al., 2019). The acids were thiopental and nifedipine (Nguyen et al., 1996; Ke et al., 2012). The neutrals were digoxin and artemether, and the zwitterion was ofloxacin (Sumner and Russell, 1976; Flor et al., 1993; Lin et al., 2016). The Schmitt and PK-Sim Standard methods recommended the use of membrane affinity $(\log M A)$ in place of $\log P$ (Willmann et al., 2005; Schmitt, 2008). Because of the limited availability of $\log M A$ values and to unify the input information for all methods, $\log P$ was used for all methods and all drugs in this investigation. The simulation scenarios for the tested drugs followed the published clinical protocols and were summarized in Supplemental Table 3.

Evaluation of PBPK Model Predictions. Model-predicted plasma concentration curves using the different calculation methods were compared with observed plasma concentrations procured from literature using WebPlotDigitizer (https://automeris.io/WebPlotDigitizer/). The percent root mean square error (RMSE) between the predicted drug plasma concentrations and the observed concentrations was defined as $R M S E=100 \frac{\sqrt{\frac{\sum_{i=1}^{n}\left(\hat{y}_{i}-y_{i}\right)^{2}}{n}}}{y_{\max }-y_{\min }}$, where $\hat{y}$ and $y$ are the predicted and observed plasma concentrations, respectively, with each containing $n$ values.

The R package PKNCA (Denney et al., 2015) was used to estimate the half-life values for the observed concentrations and each of the predicted concentration curves(). The half-life percent error was defined as

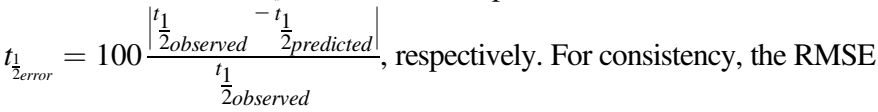
and half-life errors were reported as percentages. The mean percent RMSE and half-life percent error were calculated for each method by taking the average of the values for each drug.

\section{Results}

Verification of R Scripted Implementations of the Tissue:Plasma Partition Coefficient Prediction Methods. The R scripted implementations of each of the tissue:plasma partition coefficient prediction 


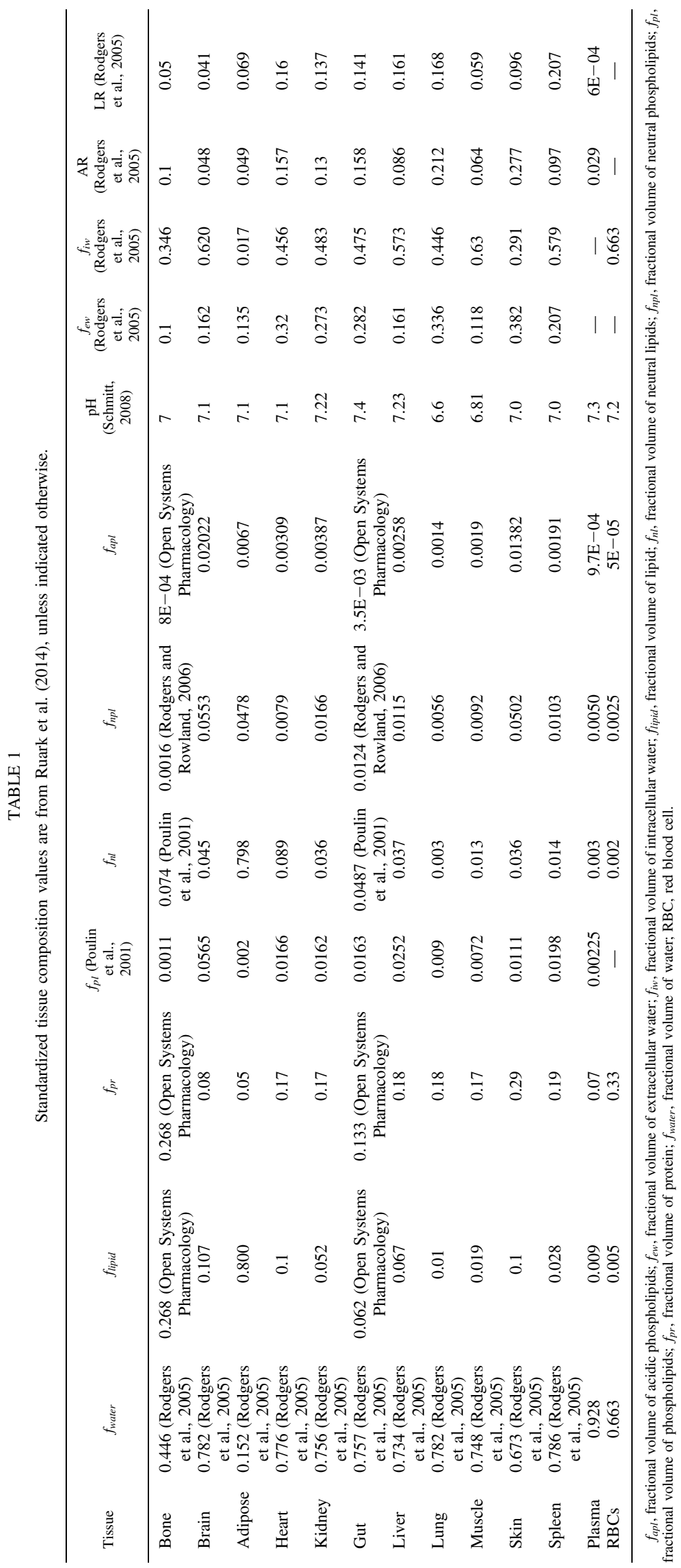

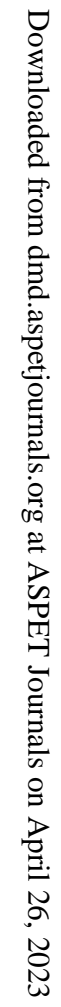


A PT

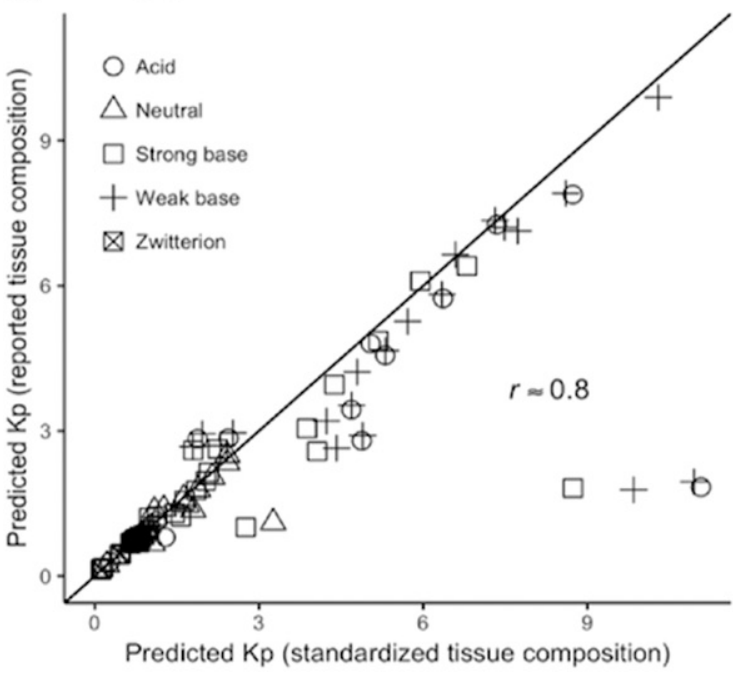

C RR

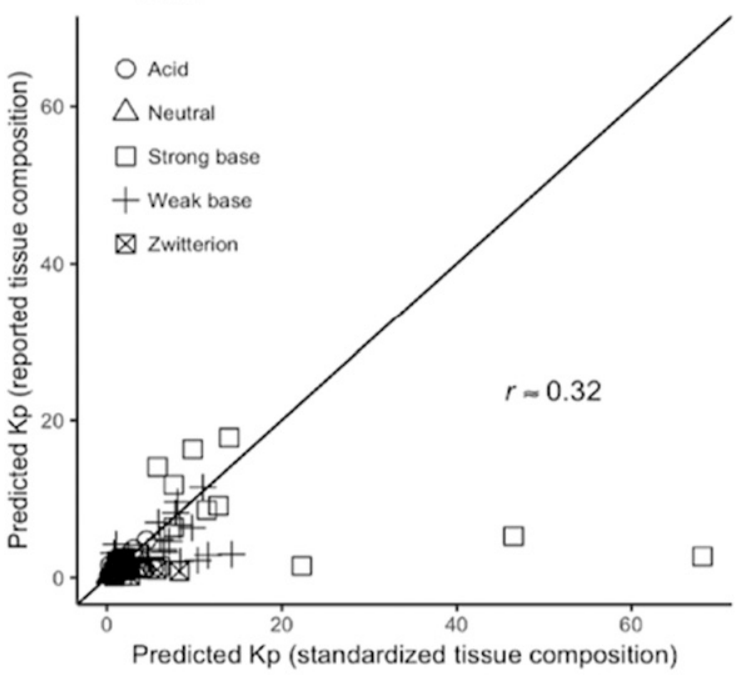

\section{E PK-Sim}

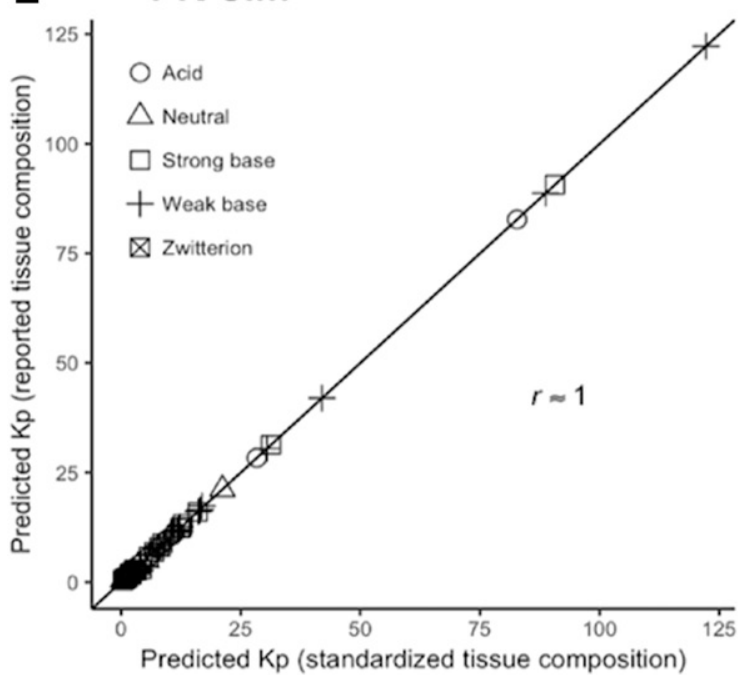

\section{B Berez}

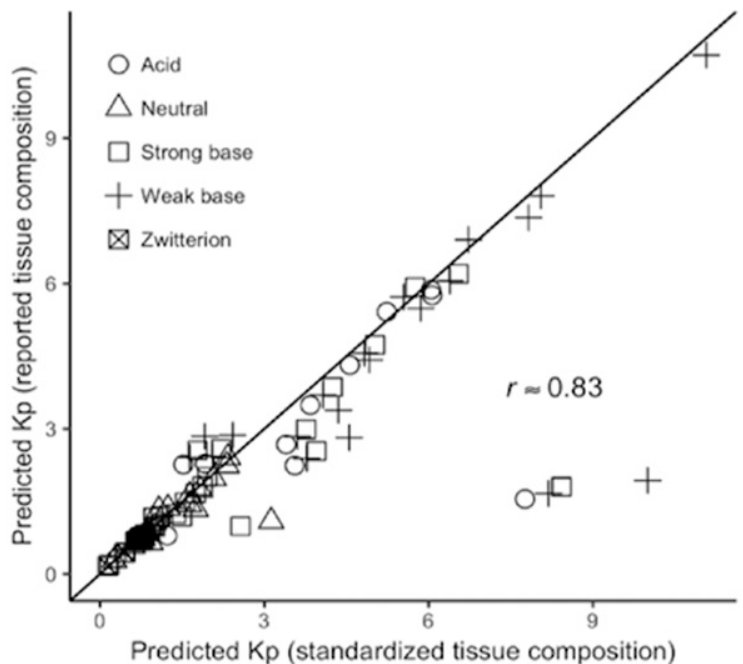

\section{Schmitt}

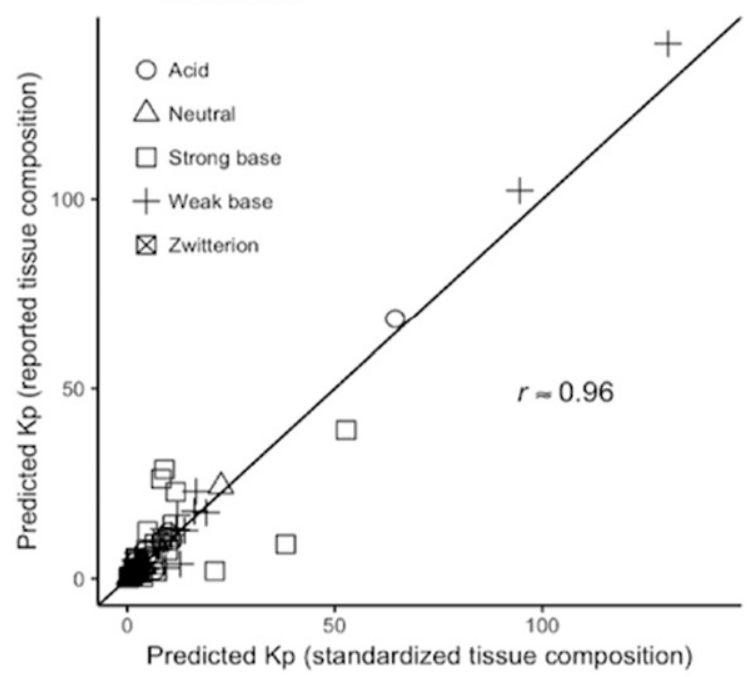

Fig. 5. Comparison between predicted tissue:plasma partition coefficients $\left(K_{p}\right)$ using the standardized tissue composition and the reported tissue compositions. The PCC for each method is denoted by $r$. (A-E) Comparisons of predictions from the PT, Berez, RR, Schmitt, and PK-Sim methods, respectively. 
A Metoprolol

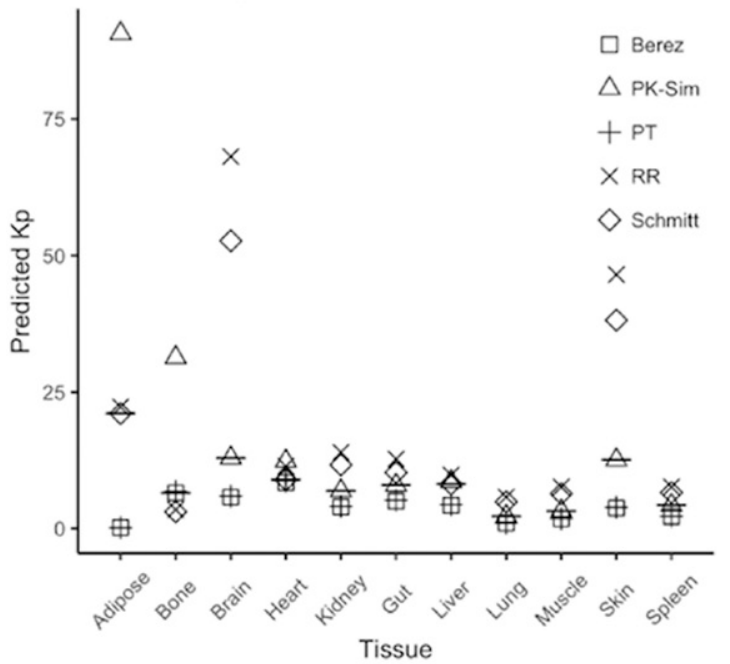

\section{B Voriconazole}

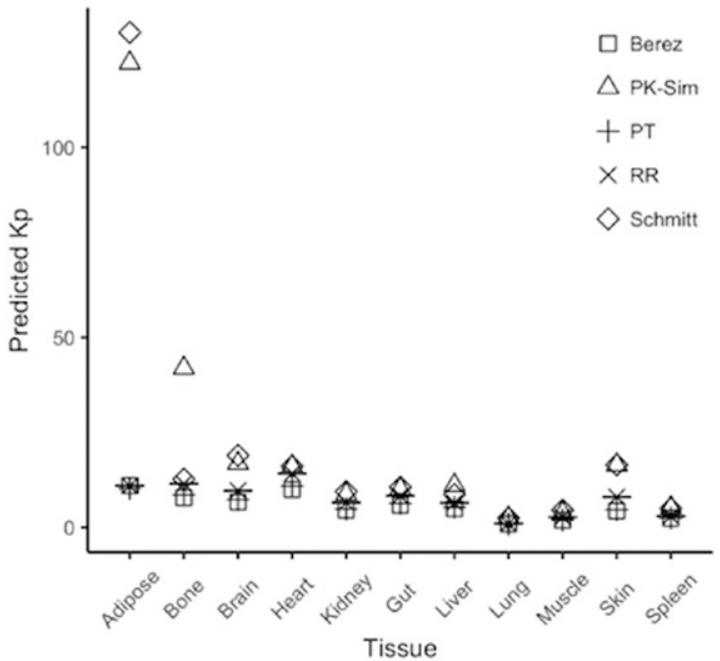

D Digoxin

\section{Nifedipine}

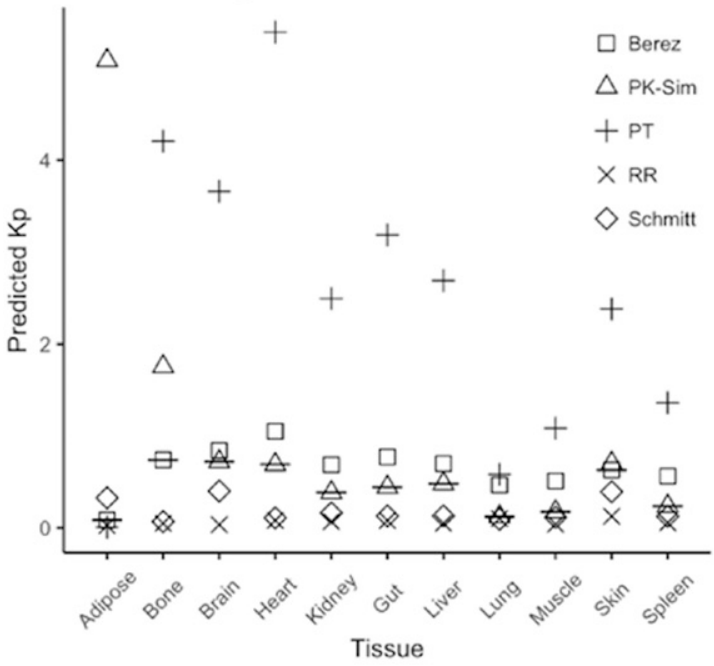

\section{E Ofloxacin}

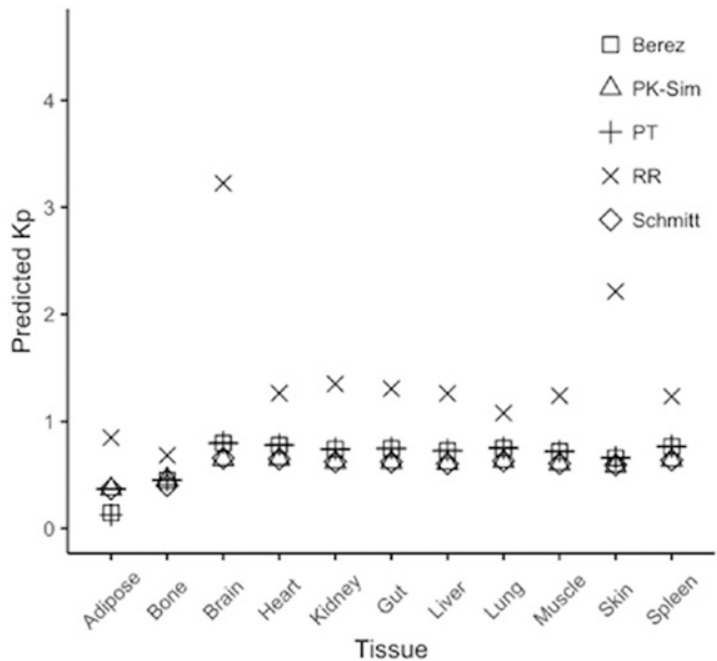

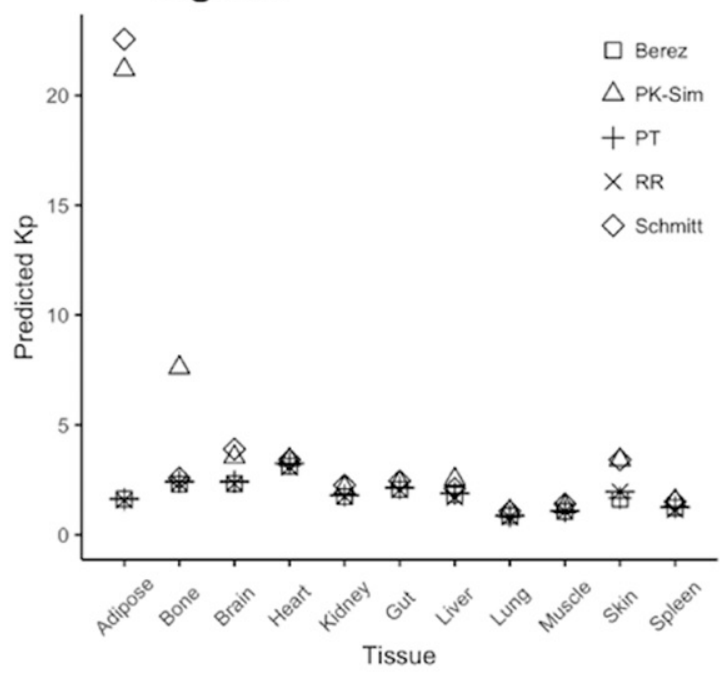

Fig. 6. Comparison of tissue:plasma partition coefficients $\left(K_{p}\right)$ predicted by each method; a representative drug from each class is considered. The horizontal bars indicate the median partition coefficient for each tissue. (A-E) Partition coefficients are compared for metoprolol (strong base), voriconazole (weak base), nifedipine (acid), digoxin (neutral), and ofloxacin (zwitterion). The PT and Berez methods predict nearly identical partition coefficients. 

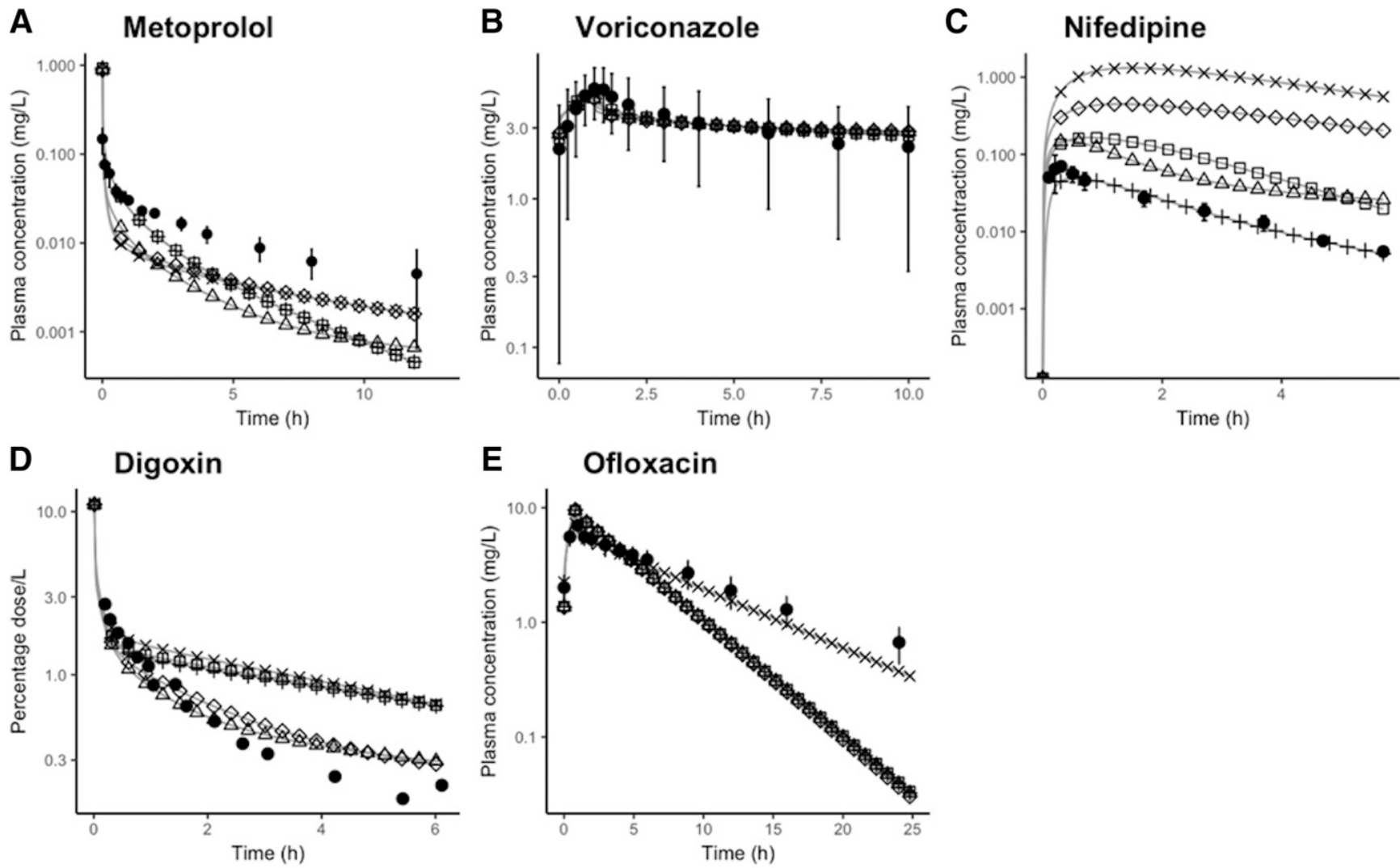

Fig. 7. Comparison between PBPK model prediction curves for each method and observed data for (A) metoprolol (10 mg, i.v.) (Gaohua et al., 2012), (B) voriconazole (4 mg/kg, i.v. infusion) (Zane and Thakker, 2014), (C) nifedipine (10 mg, by mouth) (Ke et al., 2012), (D) digoxin (0.013 mg, i.v.) (Sumner and Russell, 1976), and (E) ofloxacin (400 mg, i.v.) (Flor et al., 1993). Dots indicate means, and error bars indicate S.D. of the observed plasma concentrations.

methods were first verified against the reported partition coefficients in the respective publications (Fig. 3). The input tissue compositions were extracted from the respective publications as well, so they were different for each calculation method. The reported values that were used for verification were the PK-Sim-generated human $K_{p}$ values for Berez, Schmitt, and PK-Sim; human $V_{s s}$ values for PT; and rat $K_{p u}$ values for RR (Fig. 3). A correlation coefficient of one was found for each method, indicating that the scripts accurately reproduced the reported predictions (Fig. 3).

Impact of Different Tissue Compositions on $K_{p}$ Prediction Methods Outcome. Tissue composition information was a key input for each method, yet each method used different compositions. To demonstrate the impact of different tissue compositions on predictions, $K_{p}$ predictions from PT, Berez, Schmitt, and PK-Sim and the respective reported tissue compositions were compared against the same predictions from the RR-reported tissue composition (Fig. 4). Swapping tissue compositions had a marked impact on the predicted $K_{p}$ values from PT, Berez, and Schmitt, with PCC = 0.28, 0.26, 0.62, respectively (Fig. 3C; Fig. 4A). The PK-Sim method appeared to be more robust while predicting $K_{p}$ values from the varied tissue compositions ( $\mathrm{PCC}=0.94$, Fig. 4D).

Verification of the Standardized Tissue Composition Data Base. Table 1 contains the standardized tissue composition data base. To verify that using the standardized tissue composition yielded biologically reasonable partition coefficients, predictions from each method using the reported tissue composition were plotted against predictions from the same method for the same drugs using the standardized tissue composition (Fig. 5). PT (Fig. 5A) and Berez (Fig. 5B) produced similar predictions using the two different tissue compositions, in which the PCCs were 0.8 and 0.83 , respectively. RR had the lowest correlation ( $\mathrm{PCC}=0.32$, Fig. $5 \mathrm{C}$ ). The Schmitt method produced very similar predictions using the two tissue compositions (PCC $=0.96$, Fig. 5D), and the PK-Sim method produced seemingly identical predictions (PCC $=1$, Fig. 5E).

Quantifying the Impact of Each Tissue:Plasma Partition Coefficient Method on PBPK Model Outputs. Predictions of partition coefficients for each tissue were compared using a representative drug from each class: metoprolol (strong base), voriconazole (weak base), nifedipine (acid), digoxin (neutral), and ofloxacin (zwitterion) (Fig. 6). The PT and Berez methods predicted smaller partition coefficients for every tissue in comparison with the other methods, and adipose had the greatest difference from the median (Fig. 6A). The Schmitt and PK-Sim methods predicted the adipose partition coefficient for voriconazole to be an order of magnitude greater than the values predicted by the other methods (Fig. 6B). For nifedipine, the PT method predicted the largest partition coefficients for all tissues except adipose, whereas the RR method predicted the smallest values for all tissues (Fig. 6C). The Schmitt and PK-Sim methods had higher predictions for the adipose partition coefficient for digoxin (neutral) than the other methods (Fig. 6D). The RR method predicted the highest partition coefficients for ofloxacin (zwitterion) for all tissues (Fig. 6E). Predictions of partition coefficients were also compared for caffeine, alfentanil, midazolam, nevirapine, thiopental, and artemether (Supplemental Fig. 1). The RR method predicted the largest partition coefficients for all tissues for 
$\square$ Berez $\triangle$ PK.Sim + PT $\times$ RR $\diamond$ Schmitt

A RMSE (standardized TC)

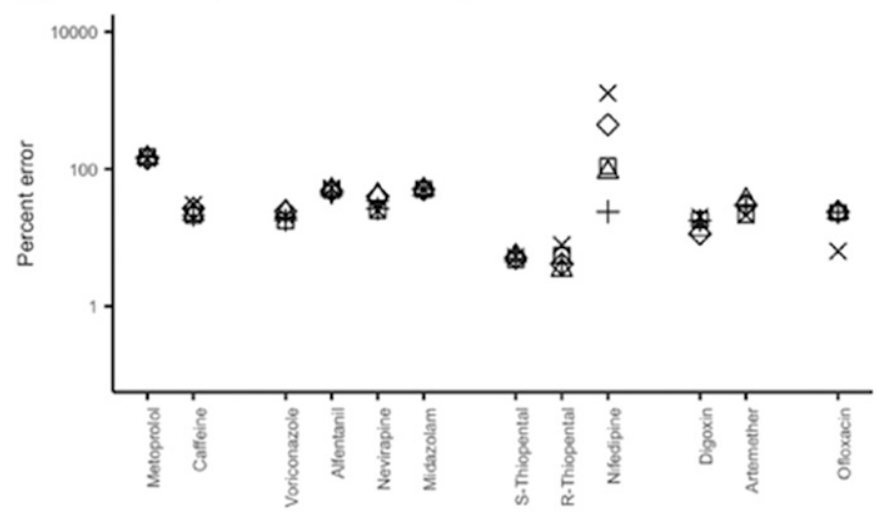

C

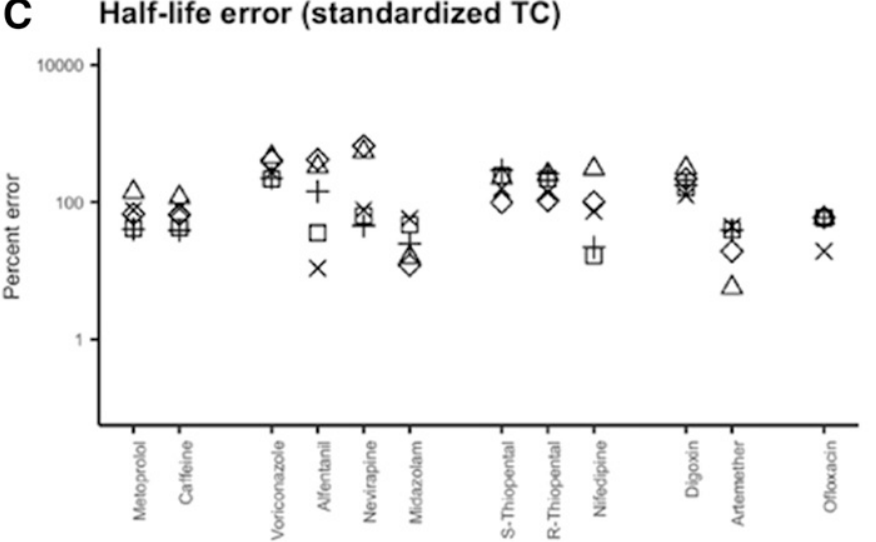

B RMSE (reported TC)

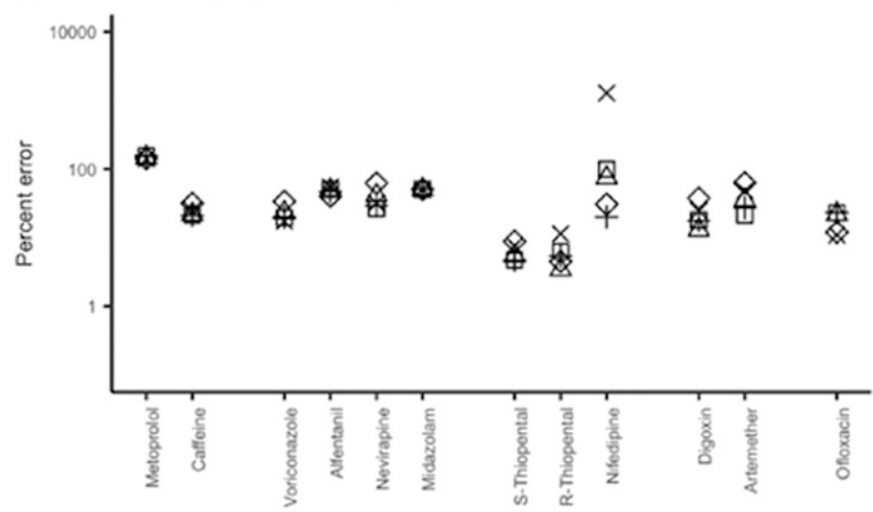

D Half-life error (reported TC)

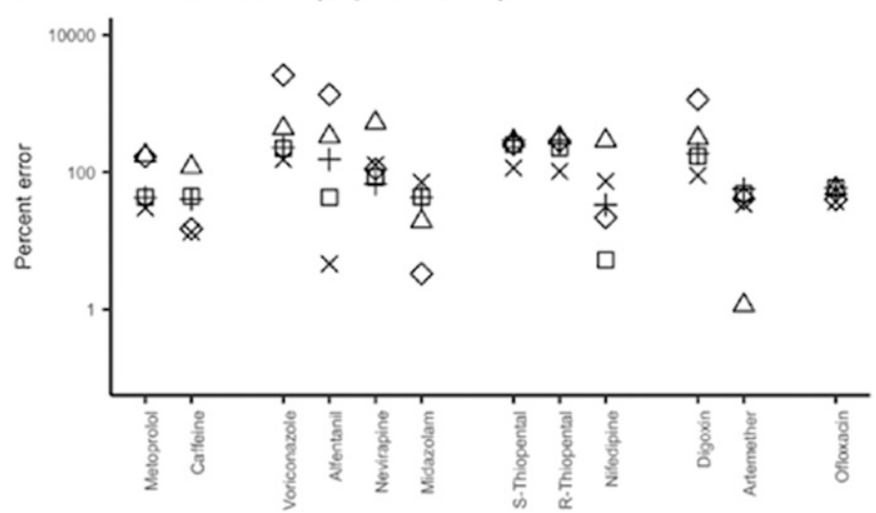

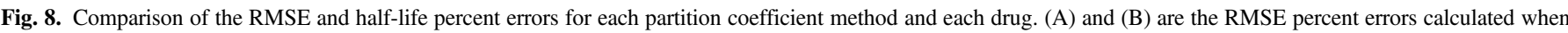

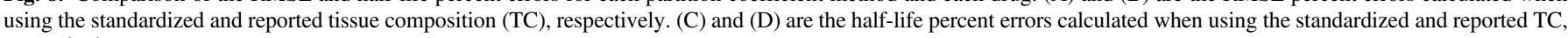
respectively.

caffeine (Supplemental Fig. 1A). For alfentanil, the PT methods predicted the largest partition coefficients for most of the tissues (Supplemental Fig. 1B). The PK-Sim and Schmitt methods had the highest predictions for the adipose partition coefficient for midazolam, nevirapine, thiopental, and artemether (Supplemental Fig. 1, B-F). Interestingly, the tissue:plasma partition coefficient for adipose was generally the most variable for every drug tested.

The PBPK model predictions for each partition coefficient method were compared with observed data for each drug (Fig. 7; Supplemental Fig. 2). Notably, no model fitting was conducted for these predictions. The PBPK model predictions using the PT and Berez methods matched the metoprolol observations more closely early in the simulated profile (e.g., prior to approximately 5 hours postdose) compared with later observations (Fig. 7A). For voriconazole, the PBPK models using each method predicted similar plasma concentration profiles (Fig. 7B). The PT method yielded a plasma concentration profile most similar to the observed data for nifedipine, whereas the RR method resulted in overprediction of the plasma concentration for the entire simulation time (Fig. 7C). The Schmitt and PK-Sim methods yielded more accurate PBPK model predictions for digoxin than the other methods (Fig. 7D). The RR method resulted in the most accurate PBPK model prediction for ofloxacin (Fig. 7E). The RR method yielded the lowest plasma concentration profile for the simulation time for caffeine (Supplemental Fig. 2A). The simulated plasma concentration profiles were similar for all methods for alfentanil, midazolam, and artemether
(Supplemental Fig. 2, B, C, and G). The Schmitt and PK-Sim methods underpredicted the plasma concentration profile for nevirapine (Supplemental Fig. 2D). The PK-Sim method yielded the plasma concentration profiles most similar to the observed data for $S$-thiopental and $R$-thiopental (Supplemental Fig. 2, E and F).

Precision and bias metrics were measured for PBPK predictions using the standardized and reported tissue compositions (Fig. 8; Table 2). The results showed that no one partition coefficient estimation method consistently outperformed the others. When using the standardized tissue composition, the RR method performed best for ofloxacin, whereas the PT method resulted in the smallest errors for nifedipine. For the other nine drugs, the results were mixed across the metrics. For example, digoxin PK prediction resulted in the smallest percent RMSE with Schmitt and the smallest half-life percent error with RR.

Generally, the estimated errors when using the reported tissue compositions were comparable to the ones estimated when using the standardized tissue compositions (Figs. 8 and 9; Table 2). The only few exceptions were mostly related to the Schmitt method, which seemed more sensitive to the tissue composition used in making the PBPK predictions than the other methods (Fig. 9).

\section{Discussion}

Tissue:plasma partition coefficients are a key component in PBPK modeling, but they are impractical to measure experimentally. The 
introduction of in silico, mechanistic-based methods to predict the coefficients was a considerable advancement in PBPK modeling; these methods alleviated the need for in vivo animal experiments to derive the partition values (Jones and Rowland-Yeo, 2013). Furthermore, mechanistic-based methods are generally based on human tissue composition, which carries a potential advantage over values experimentally determined using animal tissue composition. Since each of these methods uses different assumptions and different input information, each one can also produce different partition coefficient predictions. It was unclear whether one method was superior for a certain class of drugs or no method was consistently more accurate than the others. In the context of PBPK modeling, it was then unclear which method should be used to predict plasma concentration profiles.

This study sought to compare the PBPK model predictions generated by five commonly used partition coefficient methods with observed data. The results from swapping tissue compositions demonstrated the impact of tissue compositions on partition coefficient predictions and highlighted the importance of reaching a standardized tissue composition to eliminate this additional source of variability while quantifying the impact of these different calculation methods on PBPK model predictions. A standardized tissue composition data base was established and used in all of the partition coefficient prediction methods in the study. The standardized tissue composition provided a means for a quantitative comparison of these methods based solely on their underlying assumptions. Because of limitations in available data, values for $f_{e w}, f_{i w}, \mathrm{AR}$, and LR for all tissues and $f_{n p l}$ for bone and gut were from rats, but the flexible implementation of the standardized tissue composition data base ensures a mechanism for simple updating when the human data becomes available.

Partition coefficient predictions from each method using the reported tissue composition were compared with predictions using the standardized tissue composition. For the PT and Berez methods, the heart partition coefficient differed the most remarkably between the predictions using the reported and standardized tissue compositions. This difference likely resulted from differences between the fraction of neutral lipids in the heart in the reported and standardized tissue compositions. The RR method predictions had the lowest correlation, which was anticipated given that the reported tissue composition was measured in rats, whereas those in the standardized tissue composition were human. Further, the reported tissue compositions relevant to the Schmitt and PK-Sim methods were similar to the corresponding ones in the standardized tissue composition, so the close correlation between the predictions was expected. The results provided confidence in the $K_{p}$ predictions generated using the standardized tissue composition, and hence, the standardized tissue composition was used to calculate the partition coefficients for the remainder of this study.

Comparing the impact of the different partition coefficient calculation methods on PBPK model predictions was then carried out using the standardized tissue composition. As these calculation methods are often used prior to the availability of clinical data, the aim of the current work was not to further consider the subsequent estimation of these values from the available data. For example, the curated observed data (Fig. 7; Supplemental Fig. 2) could be further considered through sensitivity analyses and subsequent parameter optimization of $K_{p}$ following an approach similar to that recently described (Yau et al., 2020). The outcomes of such an approach, however, would be highly reliant on the collection designs employed by each of the studies used for the example drugs. Such results, if conducted subsequent to the findings reported herein, may add to our understanding of extending PBPK models in the circumstances when clinical data are available.

This study considered the performance of the partition coefficient methods for different classes of drugs. For strong bases, weak bases, 
$\square$ Berez $\triangle$ PK-Sim + PT $\times$ RR $\diamond$ Schmitt
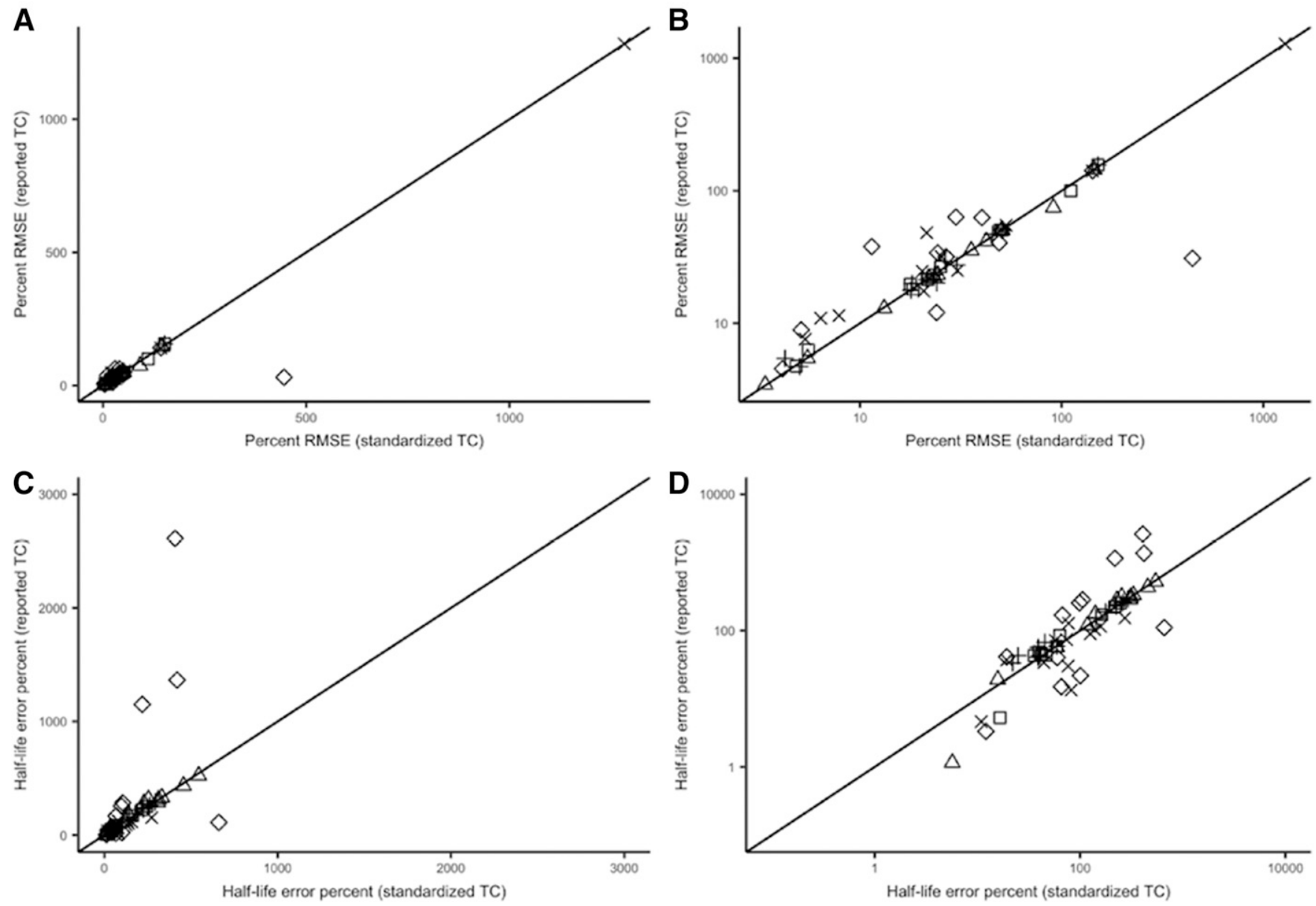

Fig. 9. Comparison of the percent RMSE (top) and half-life error percent (bottom) calculated when using the standardized and reported tissue composition (TC) on linear (A and C) and $\log (\mathrm{B}$ and $\mathrm{D})$ scales.

acids, and neutrals, no single partition coefficient method consistently produced PBPK model predictions that were more accurate than the other methods. In contrast, for zwitterions, the RR method appeared superior. However, because of the limited availability of data for zwitterionic drugs, only one drug was investigated, and, as such, further investigation is needed.

Notably, given that the individual partition coefficient calculation methods were optimized using the separate sets of reported tissue compositions, the PBPK predictions using the standardized tissue composition data base might have been biased toward some of the methods with closer agreement between their reported tissue compositions and the standardized ones. A parallel analysis to investigate the impact of this possible bias was therefore undertaken. The resulting comparability of predictive performance (Figs. 8 and 9; Table 2), regardless of method, between the standardized and original reported tissue composition values further substantiated that a universal set of these values can be used without the need to switch them out specific to each partition coefficient calculation method. That is, a universal control set of tissue composition values can be used to assess the sensitivity to the partition coefficient calculation method. Generally, the error estimates from the PBPK predictions using the standardized versus the reported tissue compositions were close, with no consistently better performance for using one of them (Figs. 8 and 9; Table 2).

Additional approaches have been developed to predict tissue:plasma partition coefficients. Endo et al. (2013) suggested a general method that used tissue composition information (fraction of storage lipids, membrane lipids, albumin, other proteins, and water), similar to the methods considered here. The partition coefficients between phases (e.g., between storage lipids and water) were predicted using polyparameter linear free-energy relationships, a type of multiple linear regression model. Unlike the other mechanistic-based methods, Freitas et al. (2015) suggested a machine-learning approach to predicting tissue:plasma partition coefficients (Endo et al., 2013). Decision tree-based regression methods were implemented to predict partition coefficients and, subsequently, the volume of distribution at steady state. Both methods seem to provide accurate predictions of tissue:plasma partition coefficients. This study investigated the most commonly used methods, but these and other methods could be included in future analyses.

The results of this study demonstrated that no method was consistently superior to the others, even within classes of drugs. This highlights the need to include the process of choosing the suitable method as part of the optimization process during PBPK model development. Using the presented $\mathrm{R}$ implementation of the five most popular calculation methods as well as the standardized tissue composition data base would make the comparison, sensitivity, and optimization steps much more accessible and, thus, could eventually be part of the PBPK model-building routine.

\section{Authorship Contributions}

Participated in research design: Utsey, Riggs, Elmokadem.

Conducted experiments: Utsey, Gastonguay, Russel, Freling, Elmokadem. 
Performed data analysis: Utsey.

Wrote or contributed to the writing of the manuscript: Utsey, Riggs, Elmokadem.

\section{References}

Arundel PA (1997) A multi-compartmental model generally applicable to physiologically-based pharmacokinetics. IFAC Proc Vol 30:129-133.

Berezhkovskiy LM (2004) Volume of distribution at steady state for a linear pharmacokinetic system with peripheral elimination. J Pharm Sci 93:1628-1640.

Björkman S, Wada DR, and Stanski DR (1998) Application of physiologic models to predict the influence of changes in body composition and blood flows on the pharmacokinetics of fentanyl and alfentanil in patients. Anesthesiology 88:657-667.

Denney WS, Duvvuri S, and Buckeridge C (2015) Simple, automatic noncompartmental analysis: the PKNCA R package. J Pharmacokinet Pharmacodyn 42:S65.

De Sousa Mendes M, Lui G, Zheng Y, Pressiat C, Hirt D, Valade E, Bouazza N, Foissac F, Blanche S, Treluyer J-M, et al. (2017) A physiologically-based pharmacokinetic model to predict human fetal exposure for a drug metabolized by several CYP450 pathways. Clin Pharmacokinet 56:537-550.

Elmokadem A, Riggs MM, and Baron KT (2019) Quantitative Systems Pharmacology and Physiologically-Based Pharmacokinetic modeling with mrgsolve: a hands-on tutorial. CPT Pharmacometrics Syst Pharmacol 8:883-893 DOI: 10.1002/psp4.12467.

Endo S, Brown TN, and Goss K-U (2013) General model for estimating partition coefficients to organisms and their tissues using the biological compositions and polyparameter linear free energy relationships. Environ Sci Technol 47:6630-6639.

Flor SC, Rogge MC, and Chow AT (1993) Bioequivalence of oral and intravenous ofloxacin after multiple-dose administration to healthy male volunteers. Antimicrob Agents Chemother 37: $1468-1472$.

Freitas AA, Limbu K, and Ghafourian T (2015) Predicting volume of distribution with decision tree-based regression methods using predicted tissue:plasma partition coefficients. J Cheminform 7:6.

Gaohua L, Abduljalil K, Jamei M, Johnson TN, and Rostami-Hodjegan A (2012) A pregnancy physiologically based pharmacokinetic (p-PBPK) model for disposition of drugs metabolized by CYP1A2, CYP2D6 and CYP3A4. Br J Clin Pharmacol 74:873-885.

Gastonguay MS, Russell S, Freling R, Riggs M, Kay K, Utsey K, and Elmokadem A (2019) Development of an open-source physiologically-based pharmacokinetic model to predict maternal-fetal exposures of CYP450-metabolized drugs. American Society for Clinical Pharmacology and Therapeutics; 2019 March 13-16; Washington, DC

Graham H, Walker M, Jones O, Yates J, Galetin A, and Aarons L (2012) Comparison of in-vivo and in-silico methods used for prediction of tissue: plasma partition coefficients in rat. J Pharm Pharmacol 64:383-396.

Jansson R, Bredberg U, and Ashton M (2008) Prediction of drug tissue to plasma concentration ratios using a measured volume of distribution in combination with lipophilicity. J Pharm Sci 97: 2324-2339.

Jones H and Rowland-Yeo K (2013) Basic concepts in physiologically based pharmacokinetic modeling in drug discovery and development. CPT Pharmacometrics Syst Pharmacol 2:e63.

Ke AB, Nallani SC, Zhao P, Rostami-Hodjegan A, and Unadkat JD (2012) A PBPK model to predict disposition of CYP3A-metabolized drugs in pregnant women: verification and discerning the site of CYP3A induction. CPT Pharmacometrics Syst Pharmacol 1:e3.
Lin W, Heimbach T, Jain JP, Awasthi R, Hamed K, Sunkara G, and He H (2016) A physiologically based pharmacokinetic model to describe artemether pharmacokinetics in adult and pediatric patients. J Pharm Sci 105:3205-3213.

Lippert Jörg, Burghaus Rolf, Edginton Andrea, Frechen Sebastian, Karlsson Mats, Kovar Andreas, Lehr Thorsten, Milligan Peter, Nock Valerie, Ramusovic Sergej, et al. (2019) Open Systems Pharmacology Community-An Open Access, Open Source, Open Science Approach to Modeling and Simulation in Pharmaceutical Sciences. CPT Pharmacometrics Syst Pharmacol 8 (12): 878-882, doi: $10.1002 / p s p 4.1247331671256$

Nguyen KT, Stephens DP, McLeish MJ, Crankshaw DP, and Morgan DJ (1996) Pharmacokinetics of thiopental and pentobarbital enantiomers after intravenous administration of racemic thiopental. Anesth Analg 83:552-558.

Poulin P, Schoenlein K, and Theil FP (2001) Prediction of adipose tissue: plasma partition coefficients for structurally unrelated drugs. J Pharm Sci 90:436-447.

Poulin P and Theil FP (2002) Prediction of pharmacokinetics prior to in vivo studies. 1. Mechanism-based prediction of volume of distribution. J Pharm Sci 91:129-156.

Poulin P and Theil F-P (2009) Development of a novel method for predicting human volume of distribution at steady-state of basic drugs and comparative assessment with existing methods. J Pharm Sci 98:4941-4961.

R Core Team (2018) R: A Language and Environment for Statistical Computing, R Foundation for Statistical Computing, Vienna, Austria.

Radić N and Prkić A (2012) Historical remarks on the Henderson-Hasselbalch equation: its advantages and limitations and a novel approach for exact $\mathrm{pH}$ calculation in buffer region. Rev Anal Chem 31:93-98.

Rodgers T, Leahy D, and Rowland M (2005) Physiologically based pharmacokinetic modeling 1: predicting the tissue distribution of moderate-to-strong bases. J Pharm Sci 94:1259-1276.

Rodgers T and Rowland M (2006) Physiologically based pharmacokinetic modelling 2: predicting the tissue distribution of acids, very weak bases, neutrals and zwitterions. J Pharm Sci 95 $1238-1257$.

Ruark CD, Hack CE, Robinson PJ, Mahle DA, and Gearhart JM (2014) Predicting passive and active tissue:plasma partition coefficients: interindividual and interspecies variability. $J$ Pharm Sci 103:2189-2198.

Schmitt W (2008) General approach for the calculation of tissue to plasma partition coefficients. Toxicol In Vitro 22:457-467.

Sumner DJ and Russell AJ (1976) Digoxin pharmacokinetics: multicompartmental analysis and its clinical implications. Br J Clin Pharmacol 3:221-229.

Willmann S, Lippert J, and Schmitt W (2005) From physicochemistry to absorption and distribution: predictive mechanistic modelling and computational tools. Expert Opin Drug Metab Toxicol 1:159-168.

Yau E, Olivares-Morales A, Gertz M, Parrott N, Darwich AS, Aarons L, and Ogungbenro K (2020) Global sensitivity analysis of the Rodgers and Rowland model for prediction of tissue: plasma partitioning coefficients: assessment of the key physiological and physicochemical factors that determine small-molecule tissue distribution. AAPS J 22:41.

Zane NR and Thakker DR (2014) A physiologically based pharmacokinetic model for voriconazole disposition predicts intestinal first-pass metabolism in children. Clin Pharmacokinet 53: $1171-1182$.

Address correspondence to: Ahmed Elmokadem, Metrum Research Group, 2 Tunxis Rd., Tariffville, CT 06081. E-mail: ahmede@metrumrg.com 